\title{
First Double-Sided End-Cap Strip Module for the ATLAS High-Luminosity Upgrade
}

\author{
Liv Wiik-Fuchs*, Ulrich Parzefall, Carlos Garcia Argos, Marc Hauser, Karl Jakobs, \\ Kambiz Mahboubi, Arturo Rodriguez Rodriguez, Frederik Ruehr \\ Albert-Ludwigs-Universität, Freiburg, Germany \\ E-mail: lwiik@mail.cern.ch
}

Dario Eliecer Ariza Alvarez, Ingo Bloch, Sergio Diez Cornell, Ingrid-Maria Gregor, Volker Prahl

DESY, Hamburg

The ATLAS Experiment will upgrade its inner tracking system for the High-Luminosity-LHC with an all-silicon system. The strip part will be based on individual modules, constructed by gluing the front-end hybrids directly onto the strip side of the sensors. These modules will then be glued onto a low-mass carbon-fibre based local support structure with integrated services. This paper presents the first double-sided module made from full-size end-cap sensors by gluing modules onto a reduced-size core. A summary on the experience gained in the prototyping process and results obtained from running this module, with emphasis on signal integrity and noise performance will be given.

Topical Workshop on Electronics for Particle Physics (TWE PP2018)

17-21 September 2018

Antwerp, Belgium

\footnotetext{
*Speaker.
} 


\section{The ATLAS Inner Tracker Upgrade}

To fully exploit the physics potential of the High-Luminosity-LHC the ATLAS experiment will replace its current inner detector with a new all-silicon Inner Tracker (ITk) [1], separated into the barrel region and end-caps at either side. The barrel region consists of 5 pixel layers, followed by 4 strip layers in the outer region. Each end-cap comprises 4 pixel rings and 6 silicon strip wheels. To accommodate the radial geometry in the end-caps the strip sensors are wedge shaped and arranged in large support structures called petals. Each wheel is constructed from 32 identical petals. The petals consist of a carbon fibre composite material with integrated cooling and services. Each petal is equipped with 9 radial symmetric sensors on either side arranged in 6 rings (R0-R5), where the innermost ring is called the R0.

\section{Ro Module Assembly}

An R0 module consists of an n-in-p type silicon sensor, two read-out hybrids and a powerboard [2]. The hybrids are made of four-layer polyimide flex circuit boards providing the necessary electrical connections and are equipped with custom-designed read-out ASICS named ABC130 (ATLAS Binary Chip) and a hybrid controller chip (HCC) both built in $130 \mathrm{~nm}$ CMOS technology [1]. The custom designed wedge-shaped silicon sensors are processed on 6-inch float-zone wafers

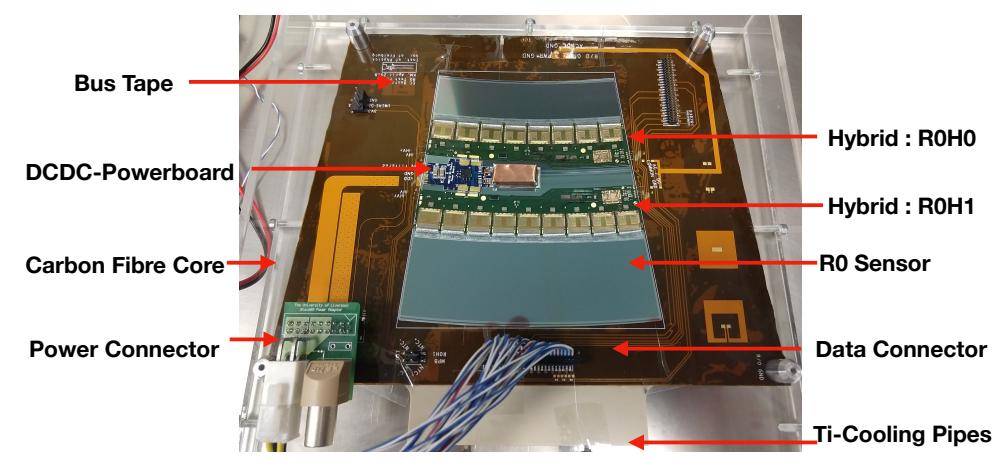

Figure 1: Topview photograph of the double-sided R0 end-cap module, glued to the bustape mounted on a petalet core.

by Hamamatsu Photonics [3]. The strips have an azimuthal orientation with a stereo rotation of 20 mrad leading to a total of $40 \mathrm{mrad}$ between the two back to back sensors. The R0 sensor contains 4352 active strips arranged in four rows with a length ranging from about $19 \mathrm{~mm}$ in the innermost row to about $32 \mathrm{~mm}$ in the outermost row. The pitch increases from $74.3 \mu \mathrm{m}$ for the inner row to $83.9 \mu \mathrm{m}$ in the outer row.

The bare hybrids are populated with $\mathrm{ABC} 130$ read-out chips and an $\mathrm{HCC}$ using an ultraviolet curing glue. To achieve the desired precision the hybrids are mounted on a vacuum jig and the glue is dispensed by a programmable glue dispenser. The ASICS are mounted using vacuumbased pick-up tools, which allows a precise positioning of the chips and a uniform glue height of $120 \pm 40 \mu \mathrm{m}$. Once the hybrids are fully equipped they are wire-bonded to testframes and electrical tests are performed. The inner hybrid is called $\mathrm{R} 0 \mathrm{H} 0$ and the outer hybrid called R0H1. They hold 
eight and nine $\mathrm{ABC} 130$ chips respectively, with 256 channels per chip. Further information on the end-cap hybrids is found in Ref. [4].

The tested hybrids are then glued directly onto the strip side of the sensor using a nonconductive epoxy glue that is cured for at least ten hours at room temperature, while the sensor is held by vacuum on another custom built precision jig. To attach the hybrids the same pick-up tools as for the hybrid population with ASICS are used for referencing heights. The sensor strips are then connected to the front-end channels on the ABC130 chip in a four row wire-bonding process. The $\mathrm{ROH} 0$ is wire-bonded to the inner two, while the $\mathrm{R} 0 \mathrm{H} 1$ is wire-bonded to the outer two strip rows. In the final assembly step a power-board is glued onto the sensor surface and wirebonded to the hybrids. An integral part of the power-board is the DC-DC converter, which steps the voltage down and such reduces the power losses on the cables by having a lower current. This does not only lead to more reliable operation given the smaller voltage drop, but also to a reduction of the material inside the tracker.

\section{Double sided Module Assembly}

The double-sided module is built from a petalet core with specially designed R0 bus tapes glued onto each side. The tested R0 modules are glued on top of the bus tapes. A topview of the assembled double-sided module is shown in Figure 1 and further information on the building process is found in Ref. [5]. The petalet is a small scale prototype petal and is made of carbon foam and carbon honeycomb material with carbon sheet facings. This sandwich construction provides an excellent heat transfer and a high structural rigidity. The petalet core holds an embedded $\mathrm{U}$ shaped titanium pipe for $\mathrm{CO}_{2}$ cooling. The R0 bus tapes, made in flex, are attached with an electronics grade epoxy glue to either side of the petalet core.

Including the bus tapes, the petalet has a total thickness of $5.4 \mathrm{~mm}$ and weighs $77 \mathrm{~g}$. The bus tapes hold all integrated services including power, control and data transmission and thus facilitate the read-out of the active modules. The individually tested modules, named FR_2 and FR_5, are then in turn glued onto the petalet using a thermally conductive and electrically insulating silicone gel. The glue pattern was optimised to achieve full coverage while keeping a minimal glue layer of about $150 \mu \mathrm{m}$. Since the hybrids are attached directly to the silicon sensor the heat path benefits from the large cross-sectional area of the sensor to the cooling within the petalet core.

\section{Electrical Tests of the Double-Sided Strip Module}

A series of analogue and digital electrical tests were performed both on the individual modules and on the fully assembled double-sided module. The test sequence includes initial powering tests, and sending a series of trigger bursts, which ensure the correct initialisation and communication with the front-end electronics. Afterwards, a strobe delay test is done, which determines the phase of the test charge injection relative to the trigger command and thus defines the optimal setting of the delay register. These are set individually for each ABC130 chip. Thereafter threshold scans are performed to define the gain, offset and linearity of the amplifiers, by measuring their response to various injected charges. The occupancy versus threshold data is measured for each channel and the resulting occupancy ranges from $0 \%$ to $100 \%$. The occupancy distribution is then fitted 

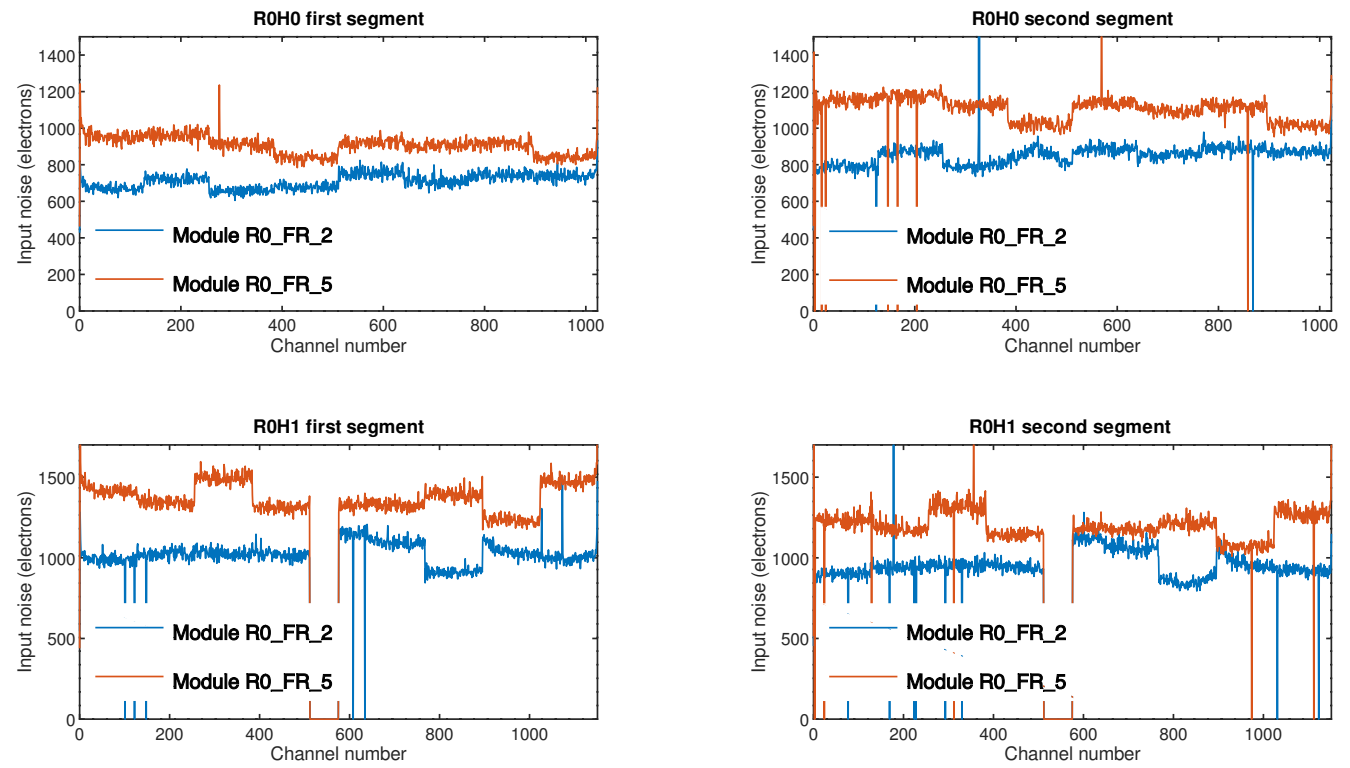

Figure 2: Equivalent Noise Charge (ENC) as a function of the channel number, shown for the FR_2 module in blue and the FR_5 in orange. The upper plots show the results of the R0H0 and the lower ones depict the R0H1.It should be noted that the 128 empty channels in the lower figures are an artefact due to the division of the data streams of the $\mathrm{R} 0 \mathrm{H} 1$ into four and five chips.

with a complementary error function, known as s-curve. These are used to extract the input noise, typically given in electrons Equivalent Noise Charge (ENC), which is determined by the standard deviation of the s-curve at that charge divided by the amplifier gain. The threshold at which the occupancy is $50 \%$ is called vt50 point and defines the mean response of the amplifier to the injected charge. This is extracted from a quadratic fit to the vt50 points as a function of the injected charge. The final test in this sequence is the noise occupancy test. It measures the noise as a function of the threshold and is determined by the number of hits returned from the front ends for increasing threshold values without any charge injection. For efficient detector operations it is necessary to keep the noise hits at a level of the order of $10^{-4}$.

The initial testing prior to assembly of the double-sided module of the FR_2 and FR_5 modules showed the expected performance and no unexpected behaviour was observed. After the assembly of the double-sided module, the electrical tests were repeated and both sides were read-out simultaneously. The equivalent noise charge of the double-sided module is shown in Figure 2, in orange for FR_5 and blue for FR_2. The double-sided module shows a flat and reasonable noise distribution. However, the noise of the FR_5 is consistently between 200 and 400 electrons higher. A noise of about 800 (1100) electrons is found for FR_2 (FR_5) in the shorter strip segments connected to the R0H0, while a noise of about 1000 (1400) electrons is measured for the FR_2 (FR_5) in the longer strip segments connected the R0H1. The differences in the noise and the unexpected large chip to chip variations are currently under further investigations, and could for instance be linked to humidity or stress induced on the sensor during the assembly or testing process.

The noise occupancy test results are shown in Figure 3 for the R0H0 hybrid on the left and 


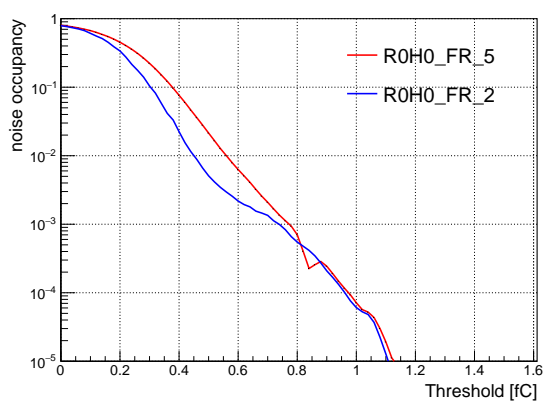

(a) $\mathrm{ROHO}$

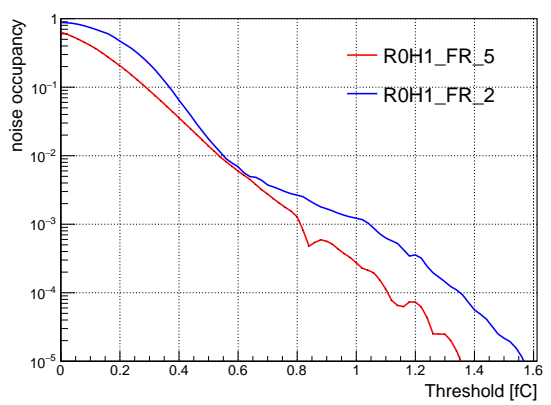

(b) $\mathrm{R} 0 \mathrm{H} 1$

Figure 3: Noise Occupancy test of the R0H0 (a) and R0H1 (b) of the double-sided module shown in red for the FR_5 and in blue for the FR_2.

the R0H1 hybrid on the right. A good noise occupancy performance is measured for the R0H0 hybrids on both modules and values below $10^{-4}$ are achieved at a threshold of $1 \mathrm{fC}$. A slightly worse performance is found for the R0H1 hybrids and a noise occupancy below $10^{-4}$ is measured at $1.1 \mathrm{fC}$ for the FR_5 module and at $1.35 \mathrm{fC}$ for the FR_2 module. The slightly unexpected shape and worse performance of the FR_2 module is connected to large common mode fluctuations, which are under current investigation.

\section{Conclusion}

The successful assembly and testing of the first double-sided end-cap module allowed a validation of the general assembly procedures. Electrical tests were performed and a simultaneous read-out of both modules was achieved. The electrical test confirmed to large extent the expected electrical performance and the module has since been measured in a beam test.

\section{Acknowledgements}

This work has been supported and financed by the German Federal Ministry of Education and Research (BMBF, Germany) and the Helmholtz Association HGF .

\section{References}

[1] ATLAS Collaboration, "Technical Design Report for the ATLAS Inner Tracker Strip Detector", CERN-LHCC-2017-005, April 2017 https : / cds . cern. ch/record/2257755

[2] "C. Garcia Argos, "Assembly and electrical tests of the first full-size forward module for the ATLAS ITk Strip detector", doi: 10.1016/j.nima.2018.09.082

[3] Hamamatsu Photonics K.K., 1126-1 Ichino-cho, Hamamatsu-shi 435-8558, Japan.

[4] Carlos Garcia Argos et. al., "Front-end Electronics of the Forward Strip Detector for the ATLAS HL-LHC Upgrade", Proceedings of this conference 2018

[5] S. Kuehn et. al., "Prototyping of petalets for the Phase-II upgrade of the silicon strip tracking detector of the ATLAS experiment", JINST 13 (2018) T03004 doi: 10.1088/1748-0221/13/03/T03004 